\title{
An Analysis of Preanalytical Errors and Turnaround Time in Emergency Biochemistry Laboratory in a Tertiary Care Hospital in New Delhi
}

\author{
Namrata Bhutani ${ }^{1}$, Neha Bhutani ${ }^{2}$ \\ ${ }^{1}$ Department of Biochemistry, Vardhaman Mahavir Medical College and Safdarjung Hospital, \\ New Delhi, India. ${ }^{2}$ ESIC Dental College, Rohini, New Delhi, India.
}

\section{ABSTRACT}

\section{BACKGROUND}

In modern diagnostics, it is extremely important to maintain and ensure quality of laboratory results dispatched. It is part of the total quality management and an essential criterion for accreditation of the laboratory. The analysis of biochemistry samples can be broadly divided into three phases: pre-analytical, analytical and post-analytical phase. We wanted to identify the commonly occurring pre-analytical errors and determine the turnaround time in the emergency biochemistry laboratory at a tertiary care hospital in Delhi.

\section{METHODS}

A cross-sectional study was done on a total of 2,73,111 samples received in the emergency biochemistry laboratory from September 2018 to August 2019 and an analysis of occurrence of pre-analytical errors was done, retrospectively. Additionally, the turnaround time of the laboratory was evaluated over a period of two months from July 2019 to August 2019 and average time was recorded. Data was collected from entry registers and rejected samples registers.

\section{RESULTS}

In this study it was found that $10.58 \%$ of the total samples received were rejected. Moreover, overall turnaround time was found to be 108 minutes (median value). In the present study, haemolysis was the most common reason for sample rejection. (63.14\% of total rejections). Additionally, the second most common error was inadequate samples. 6570 samples were rejected due to this reason (22.73\%).

\section{CONCLUSIONS}

Haemolysis was the most common cause of rejection in the emergency biochemistry laboratory. Also, it was seen that the most time-consuming step was analysis in auto-analyser with respect to contribution to turnaround time. As a matter of fact, Pre-analytical errors can adversely affect the treatment of patients. However, most of the errors can be reduced by proper training of the staff and checking competency thoroughly by conduction of practical and theory assessment of laboratory personnel at frequent intervals.

\section{KEY WORDS}

Pre-Analytical Errors, Turnaround Time, Sample Rejection, Clinical, Biochemistry, Emergency Laboratory, Haemolysis.
Corresponding Author: Dr. Namrata Bhutani, D-162, Tagore Garden Extension, New Delhi-110027, India. E-mail: namrata.bhutani@gmail.com

DOI: $10.14260 /$ jemds/2020/229

Financial or Other Competing Interests: None.

How to Cite This Article:

Bhutani N, Bhutani N. An analysis of preanalytical errors and turnaround time in emergency biochemistry laboratory in a tertiary care hospital in New Delhi. J. Evolution Med. Dent. Sci. 2020;9(13): 1065-1068 DOI:
Submission 31-12-2019,

Peer Review 10-03-2020

Acceptance 16-03-2020,

Published 30-03-2020. 


\section{BACKGROUND}

Medical biochemistry laboratories play an important role in modern-day diagnosis. Therefore, more importance is given to ensure the quality of laboratory tests. The errors in laboratory are classified into pre-analytical, analytical and post-analytical phase depending on the time of presentation. As per current literature, most of the laboratory errors are either pre-analytical (46-48\%) or post-analytical (18-47\%) whereas $7-13 \%$ errors occur in the analytical phase. ${ }^{1}$ Also, the pre-analytical phase is the most significant and difficult to control and maintain because a lot of professionals are involved in this phase. ${ }^{1}$ Moreover, they are rarely covered by quality control programs. International federation of clinical chemistry and laboratory medicine working group for laboratory errors and patient safety (IFCC-WG-LEPS) has highlighted the most common pre-analytical errors in laboratory practice. ${ }^{2}$ In today's times, clinical laboratories have an important role in the diagnosis and treatment of patients because they supply data from the analysis of body fluids. However, inaccurate laboratory results can cause a number of clinical problems including delayed clinical diagnosis, further laboratory testing, and incorrect diagnosis and management. As stated previously, preanalytical errors account for approximately $60 \%-70 \%$ of laboratory errors. Out of these, the main causes of preanalytical errors are inappropriate test request, patient preparation, specimen collection, specimen transportation, and specimen preparation for testing. Therefore, decrease in error rates in preanalytical phase is imperative to ensure cost effectiveness, patient satisfaction and quality of laboratory service. Chemical pathologist and biochemists plays a vital role in modern day diagnosis and treatment. Therefore, it is of paramount importance that results which are generated in the laboratory are accurate and precise as clinical management depends on them. The procedure of analysis of biochemistry samples is comprised of 3 phases, namely, Preanalytical, Analytical and Post analytical. The preanalytical phase consists of processes that take place from the time a laboratory request is made by a physician until the sample is ready for testing. Thus, it includes ordering of test by clinician, filling up of request forms, collection of samples, sample transportation, and finally sample preparation for analysis. The analytical phase comprises of analysis of samples in the auto-analyser and generation of reports. Moreover, the post-analytical phase comprises of communication of laboratory reports to clinicians for appropriate clinical management of patient. Needless to say, errors at any of these phases can seriously affect the correct diagnosis and overall well-being of the patient. However, with automation of most of the laboratories, time for analysis as well as laboratory errors have significantly declined, more specifically for those that occur in the analytical phase. As stated above, the maximum percentage of total errors in the entire diagnostic analysis occur in pre-analytical phase. According to various previously conducted studies, the frequency of errors in the pre-analytical phase have been reported to be $77.1 \%, 81 \%$ and $31.6-75 \%$. Since, errors can occur in any of the multiple steps involved in the Preanalytical phase it is imperative to catch the errors occurring during the pre-analytical phase. Itself. However, this is not always possible and occasionally these are detected in the analytical or postanalytical phases. Thus, although, analytical errors have reduced considerably, however a large proportion of pre-analytical errors occur and negatively impact the total error rate and subsequently reduce the accuracy of reported laboratory test results. The turnaround time (TAT) used in hospitals to assay the performance of laboratory is defined as the time taken from order entry to result reporting. Therefore, reduced turnaround time is important for early diagnosis and treatment and thus shortens patients stay in the hospital and eventually leads to increase in their satisfaction and safety. The present study was undertaken to study the prevalence and types of preanalytical errors in the clinical biochemistry emergency laboratory at a tertiary care hospital in New Delhi, India. In addition to this, the preanalytical turnaround time of emergency samples for quality and timeliness of reporting the test results was studied.

\section{METHODS}

This cross-sectional study was conducted at the Biochemistry laboratory of new emergency building of Safdarjung Hospital. This laboratory receives blood samples from all the emergency wards as well as non-routine samples for inpatient patients from rest of the wards of the hospital. Rejected samples in the emergency laboratory from September 2018 to August 2019 were reviewed retrospectively. Data was collected from entry registers and rejected samples registers.

Also, the Turnaround time (TAT) of emergency biochemistry samples were evaluated over a period of two months from July 2019 to August 2019 and total of 48,031 samples were studied. The time intervals between the stages of workflow (from receiving samples till dispatch of reports) were recorded. All samples from respective wards reach the receiving counter from where they are sent to the biochemistry lab. Samples are then sorted, centrifuged and analysed on Beckman Coulter Olympus AU 480. Reporting is done on the respective requisition forms, data entered in the sample register, reports signed and validated by the duty doctor and finally reports are sent for dispatch to the respective wards. Parameters done on blood samples in emergency laboratory include glucose, urea, creatinine, total bilirubin, direct bilirubin, ALP, ALT, AST, amylase, sodium, potassium, chloride, creatine kinase, creatine kinase-MB and lipase.

\section{Statistical Analysis}

Statistical analysis was conducted using Microsoft Excel 2016 program. Calculations of rejected specimens of biochemistry lab was presented as number and percentage. Data of TAT was expressed as median and range.

\section{RESULTS}

Out of total of 2,73,111 samples received in the laboratory, 28,904 were rejected owing to various reasons including quantity not sufficient, haemolyzed samples, lipemic samples, labelling errors, inappropriate tube, clotted or contaminated 
samples, sample mix-ups etc. as shown in table 1. This comprises of $10.58 \%$ of the total samples. As can be seen in the table, 18,250 samples were rejected due to haemolysis, which is most common cause of rejection in this study and having a rejection frequency of $63.14 \%$. Moreover, insufficient sample quantity was the next most common cause, having a frequency of $22.73 \%$. Additionally, labelling errors contributed towards $6.31 \%$ of the total samples rejected.

\begin{tabular}{|c|c|c|c|}
\hline Sl. No. & Rejection Criteria & No. of Samples, $n$ & Frequency ( \%) \\
\hline 1. & $\begin{array}{l}\text { Quantity not sufficient } \\
\text { (QNS) }\end{array}$ & 6,570 & 22.73 \\
\hline 2. & Haemolysed samples & 18,250 & 63.14 \\
\hline 3. & Lipemic samples & 03 & 0.01 \\
\hline 4. & Labelling errors & 1,825 & 6.31 \\
\hline 5. & Inappropriate tube & 365 & 1.26 \\
\hline 6. & Test not done/ available & 10 & 0.03 \\
\hline 7. & Clotted samples & 348 & 1.20 \\
\hline 8. & Sample contaminated & 730 & 2.52 \\
\hline 9. & Sample mix ups & 803 & 2.77 \\
\hline & tal samples rejected & 28,904 & 10.58 (total rejection rate) \\
\hline Tot & tal samples received & $2,73,111$ & - \\
\hline
\end{tabular}

In order to study the turnaround time in the laboratory, the time taken for complete sample processing from receiving samples in the lab till dispatch of reports was recorded for a total of 48, 031 samples as shown in table 2 . As can be seen in table 2, the analytical period took maximum time and comprised of around $42.59 \%$ of the total turnaround time, with median time for analytical period as 46 minutes. Moreover, time taken from sample receiving till loading of samples in the autoanalyzer was thirty three minutes and contributed around $30.55 \%$ to the turnaround time. Additionally, time taken from reporting till dispatch of reports was 29 minutes and contributed $26.85 \%$ of the total turnaround time. This data is presented below in table 2 .

\begin{tabular}{|ccccc|}
\hline $\begin{array}{c}\text { Steps } \\
\text { Involved }\end{array}$ & $\begin{array}{c}\text { Sample } \\
\text { Received }- \\
\text { Loading }\end{array}$ & $\begin{array}{c}\text { Analytical } \\
\text { Period }\end{array}$ & $\begin{array}{c}\text { Reporting - } \\
\text { Dispatch of } \\
\text { Reports }\end{array}$ & $\begin{array}{c}\text { Total } \\
\text { TAT } \\
\text { (Minutes) }\end{array}$ \\
$\begin{array}{c}\text { Average Time } \\
\text { taken in Minutes } \\
\text { (n=48,031) }\end{array}$ & $33 *(18-45)$ & $46 *(20-54)$ & $29 *(10-45)$ & 108 \\
$\%$ of Each Stage & $30.55 \%$ & $42.59 \%$ & $26.85 \%$ & $100 \%$ \\
\hline
\end{tabular}

Table 2. Time Taken to Complete Different Steps of Sample Processing

in Autoanalyser Expressed as Median and Range; *Median Value

\section{DISCUSSION}

In the modern diagnostic laboratories, errors are more commonly seen in pre- and post-analytical phases than in the analytical phase because these phases are not in direct control of laboratory personnel and also these phases involve multiple steps and laboratory personnel. ${ }^{3}$ Moreover, majority of the pre-analytical errors are preventable. ${ }^{4,5,6}$ In the current study, the incidence of the rejected specimens in the emergency biochemistry was $10.58 \%$. However, previous studies have reported the incidence of biochemistry samples ranging from $0.3 \%$ to $6 \% \cdot 7,8,9,10,11$ In the present study, haemolysis was the most common reason for sample rejection (63.14\% of total rejections). It is to be noted that Gokhan $\mathrm{C}^{11}$ has reported incidence of haemolysis related rejection as $74.1 \%$. Also, similar findings were found by Goswami et al ${ }^{12}$ ( $81 \%$ of total rejections). Additionally, Arul et al ${ }^{10}$ reported incidence of haemolysis as $0.03 \%$. There can be various causes of haemolysis including using a needle that is too small, pulling the syringe plunger too fast, shaking the tube vigorously, or centrifuging the sample before clot formation. ${ }^{13}$ It also leads to increased turnaround time as fresh sampling is required, mostly. ${ }^{10}$

The second most common error was inadequate samples. 6570 samples were rejected due to this reason $(22.73 \%)$. As per available literature, insufficient samples can be accounted from paediatric, neonatal and oncology wards, as peripheral vascular access is difficult.14,15,16 Incorrect phlebotomy practices due to ignorance or increased workload could be another reason. ${ }^{14}$ Incidence of misidentifications, labelling errors, incorrect vials, sample contaminated, sample mix-ups were observed to be $6.31 \%, 1.26 \%, 2.52 \%$ and $2.77 \%$ respectively. This can be attributed to excessive work-load due to large number of patients or sampling done by untrained staff. ${ }^{17}$ Incidence of test not done/available was $2.52 \%$, which could be because of lack of information in the wards.

Moreover, the incidence of clotted samples was found to be $1.2 \%$.The chief reason could be improper handling of blood samples including poor mixing, keeping at horizontal position. However, Gokhan $\mathrm{C}^{11}$ reported incidence of clotted samples to be $45.6 \%$ in haematology laboratory. Also, Arul et $\mathrm{al}^{10}$ reported that $0.12 \%$ samples were clotted in biochemistry laboratory. Since, pre-analytical errors can adversely affect treatment of patients it is essential to control. One way to control and minimize these errors is proper training as most of the errors can be reduced by proper training of the staff and checking competency through by conduction of practical and theory assessment at frequent intervals. ${ }^{10,18}$

\section{Limitations}

The limitations of the present study include that for the purpose of the research, only samples received in emergency were included and routine samples were not included at all in the study. Thus the results obtained could not be representative of the overall picture in the biochemistry laboratory. Also, no comparison was made between day, evening and night shifts samples in the emergency laboratory. Since, the number of samples as well as the efficiency of personnel varies in different shifts, this factor is important and could not be considered in this study.

\section{CONCLUSIONS}

In the current study, incidence of pre-analytical errors was found to be $10.58 \%$ in the emergency lab. Moreover, haemolysis of blood samples was the most common cause of rejection. Also, the total turnaround time (median value) was found to be 108 minutes and the time taken for analysis in the auto-analyser was the main contributing factor towards turnaround time. Therefore, it is recommended to avoid these errors, and conducting of adequate and continuous training of hospital staff including lab personnel. 


\section{REFERENCES}

[1] Cornes MP, Atherton J, Pourmahram G, et al. Monitoring and reporting of preanalytical errors in laboratory medicine: the UK situation. Ann Clin Biochem 2016;53(Pt 2):279-84.

[2] Sciacovelli L, Plebani M. The IFCC Working Group on laboratory errors and patient safety. Clin Chim Acta 2009;404(1):79-85.

[3] Mohammedsaleh ZM, Mohammedsaleh F. A review article of the reduce errors in medical laboratories. Glob J Health Sci 2015;7(1):46-51.

[4] Carraro P, Plebani M. Errors in a stat laboratory: types and frequencies 10 years later. Clin Chem 2007;53(7):1338-42.

[5] Kalra J. Medical errors: impact on clinical laboratories and other critical areas. Clin Biochem 2004;37(12):105262.

[6] Astion ML, Shojania KG, Hamill TR, et al. Classifying laboratory incident reports to identify problems that jeopardize patient safety. Am J Clin Pathol 2003;120(1):18-26.

[7] Dale JC, Novis DA. Outpatient phlebotomy success and reasons for specimen rejection. Arch Pathol Lab Med 2002;126(4):416-9.

[8] Guimaraes AC, Wolfart M, Brisolara MLL, et al. Causes of rejection of blood samples handled in the clinical laboratory of a University Hospital in Porto Alegre. Clin Biochem 2012;45(1-2):123-6.

[9] Lay SI, Pinar A, Akbiyik F. Classification of reasons for rejection of biological specimens based on pre-analytical processes to identify quality indicators at a university hospital clinical laboratory in Turkey. Clin Biochem 2014;47(12):1002-5.
[10] Arul P, Pushparaj M, Pandian K, et al. Prevalence and types of preanalytical error in hematology laboratory of a tertiary care hospital in South India. J Lab Physicians 2018;10(2):237-40.

[11] Cakirca G. The evaluation of error types and turnaround time of preanalytical phase in biochemistry and hematology laboratories. Iran J Pathol 2018;13(2):173-8.

[12] Goswami B, Singh B, Chawla R, et al. Evaluation of errors in a clinical laboratory: a one-year experience. Clin Chem Lab Med 2010;48(1):63-6.

[13] Carraro P, Servidio G, Plebani M. haemolyzed specimens: a reason for rejection or a clinical challenge? Clin Chem 2000;46(2):306-7.

[14] Chawla R, Goswami V, Tayal D, et al. Identification of the types of preanalytical errors in the clinical chemistry laboratory: 1-year study of G.B. Pant hospital. Lab Med 2010;41(2):89-92.

[15] Detaille T, Pirotte T, Veyckemans F. Vascular access in the neonate. Best Pract Res Clin Anaesthesiol 2010;24(3):403-18.

[16] Gallieni M, Pittiruti M, Biffi R. Vascular access in oncology patients. CA Cancer J Clin 2008;58(6):323-46.

[17] Dikmen ZG, Pinar A, Akbiyik F. Specimen rejection in laboratory medicine: necessary for patient safety? Biochem Med (Zagreb) 2015;25(3):377-85.

[18] Najat D. Prevalence of pre-analytical errors in clinical chemistry diagnostic labs in Sulaimani city of Iraqi Kurdistan. PLoS One 2017;12(1):0170211. 